\title{
Erratum to: Zinc modulates copper coordination mode in prion protein octa-repeat subdomains
}

\author{
Francesco Stellato $\cdot$ Ann Spevacek • \\ Olivier Proux · Velia Minicozzi · Glenn Millhauser • \\ Silvia Morante
}

Published online: 3 July 2012

(c) European Biophysical Societies' Association 2012

\section{Erratum to: Eur Biophys J (2011) 40:1259-1270 \\ DOI 10.1007/s00249-011-0713-4}

In Fig. 2 of the paper, we wanted to show a comparison among XANES spectra collected at the $\mathrm{Cu}$ K-edge of samples B_Cu, $S_{1}, S_{2}$ and $S_{3}$, as correctly stated in the figure caption. However, due to a mistake in the proofreading phase, the Fig. 2 that is present in the paper is actually a copy of Fig. 5. It, therefore, shows the comparison among EXAFS spectra at $\mathrm{Zn}$ K-edge of samples B_Zn, $S_{1 \_} Z n, S_{2} Z$ Zn and $S_{3 \_} Z n$.

The correct version of Fig. 2 is as follows:

The online version of the original article can be found under doi:10.1007/s00249-011-0713-4.

F. Stellato $\cdot$ V. Minicozzi $\cdot$ S. Morante $(\bowtie)$

Dipartimento di Fisica, Università di Roma 'Tor Vergata',

Via della Ricerca Scientifica, 1, 00133 Rome, Italy

e-mail: morante@roma2.infn.it

F. Stellato

Centre for Free-Electron Laser Science, DESY,

Notkestrasse 85, 22607 Hamburg, Germany

A. Spevacek - G. Millhauser

Department of Chemistry and Biochemistry,

University of California, Santa Cruz, CA 95064, USA

O. Proux

Observatoire des Sciences de l'Univers de Grenoble,

CNRS and Université Joseph Fourier, BP 53,

38041 Grenoble Cedex 9, France

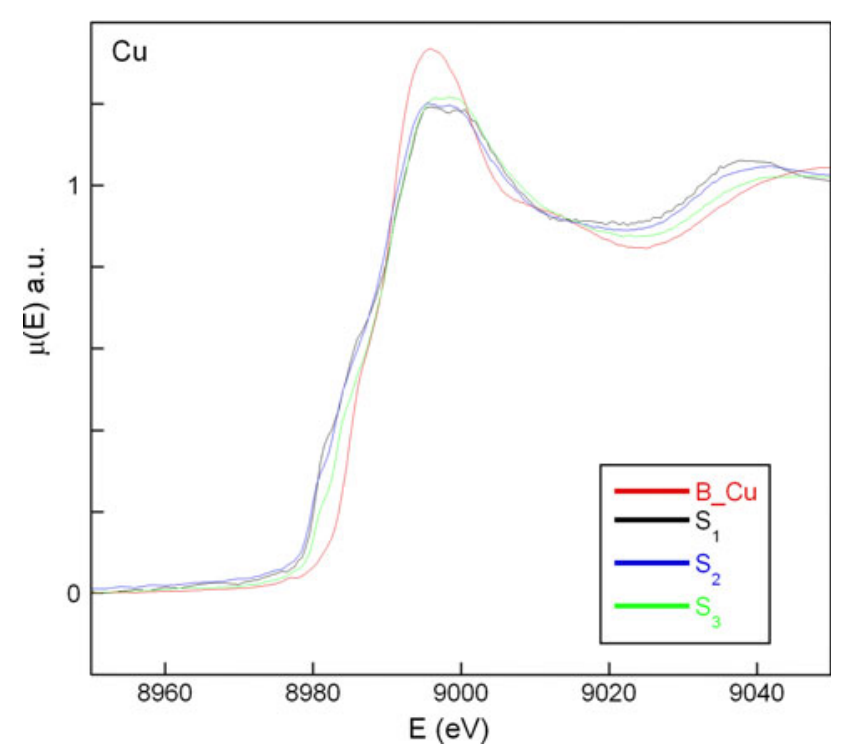

Fig. 2 Comparison among XANES spectra taken at the $\mathrm{Cu}$ K-edge of the three samples prepared at the three different $\mathrm{Cu}^{2+}$ concentrations of Table 1, in the absence of $\mathrm{Zn}^{2+}: \mathrm{S}_{1}$ (black), $\mathrm{S}_{2}$ (blue), $\mathrm{S}_{3}$ (green). The spectrum of the B_Cu buffer (red) is also shown 